\title{
Síndromes funcionais somáticas
}

\section{Functionall somatic syndromes}

\author{
Luís Augusto Pilan', Isabela M. Benseñor ${ }^{2}$
}

\begin{abstract}
Pilan LA, Benseñor IM. Síndromes funcionais somáticas. Rev Med (São Paulo). 2008 out.dez.;87(4):238-44.

RESUMO: As síndromes funcionais somáticas são muito freqüentes na prática clínica e estão na fronteira entre a clínica e a psiquiatria. Embora não sejam doenças graves, comprometem a qualidade de vida e sobrecarregam os gastos no sistema de saúde. Embora muito prevalentes, ainda geram polêmica em relação ao diagnóstico e tratamento.
\end{abstract}

DESCRITORES: Qualidade de vida. Transtornos psicofisiológicos.

\section{INTRODUÇÃO}

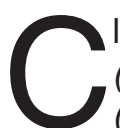

lassicamente, síndromes funcionais (SF) ou síndromes somáticas funcionais (SSF) são quadros apresentados por pacientes que se queixam de sintomas físicos cuja origem biológica não é clara na ausência de marcadores biológicos de doença ${ }^{8}$. Os sintomas inexplicáveis são alterações funcionais sem alteração da estrutura concomitante ${ }^{11}$. Muitos dos pacientes com esses diagnósticos são usuários freqüentes dos serviços de saúde na atenção primária e/ou especializada, com grande número de exames solicitados em várias investigações inconclusivas e geralmente negativas. Em alguns casos, houve indicação prévia de cirurgias sem achados conclusivos. Mesmo quando há alguma base clínica para o quadro, ela não explica a natureza e a extensão dos sintomas, nem a angústia e preocupação do paciente. Mesmo quando é evidente a relação entre os sintomas e conflitos emocionais ou eventos estressantes, o paciente mostra-se resistente em aceitar o diagnóstico de síndrome funcional somática.

Os pacientes buscam os médicos por causa dos sintomas e os médicos, baseados nos sintomas, diagnosticam doenças que tentam explicá-los. Os sintomas são experiências subjetivas que o paciente conta. A dificuldade surge quando os médicos não encontram sinais objetivos de doença. Nesse caso os sintomas são chamados de funcionais ou inexplicáveis ${ }^{20}$. Entretanto, vários são os nomes utilizados para descrever esses sintomas inexplicáveis, incluindo somatização, transtornos somatoformes, sintomas sem explicação médica e, ainda, sintomas funcionais somáticos. Um sintoma funcional somático seria aquele que, mesmo após avaliação médica, não poderia ser encaixado como parte de uma doença classicamente definida ${ }^{16,20}$.

A partir da década de 80 , a clínica médica e a psiquiatria começaram a reconhecer a existência e o desafio no diagnóstico e tratamento dessas síndromes, e os primeiros estudos sobre as síndromes funcionais somáticas mais conhecidas demonstraram a existência de extensa psicopatologia nesses pacientes ${ }^{8,9,14,15}$. A maior parte dos estudos sobre as síndromes funcionais se refere a um conjunto especifico de sintomas que caracteriza uma das síndromes. Praticamente todas as especialidades médicas apresentam suas síndromes funcionais, como a síndrome do intestino irritável (gastroenterologia), dor pélvica crônica (ginecologia),

\footnotetext{
1. Médico Assistente do Hospital das Clínicas da Faculdade de Medicina da Universidade de São Paulo - HCFMUSP.

2. Professora Associada Faculdade de Medicina da Universidade de São Paulo - FMUSP.

Endereço para correspondência: Hospital Universitário. Av. Lineu Prestes 2565. 3ํo andar - Centro de Pesquisa Clínica. CEP - 05508-000. São Paulo, SP. E-mail: isabensenor@hu.usp.br
} 
fibromialgia (reumatologia), dor torácica não cardíaca (cardiologia) e cefaléia tensional (neurologia). A lista de quais síndromes contemporâneas poderiam ser chamadas 'funcionais' é variável e controversa, e provavelmente em grande parte destes diagnósticos coexistem causas orgânicas e psicossociais, muitas vezes nos mesmos indivíduos. O Quadro 1 lista as síndromes funcionais somáticas mais conhecidas ${ }^{20}$. Algumas, como a dispepsia funcional não ulcerosa e a cefaléia do tipo tensional, são muito freqüentes na prática clínica, enquanto outras são bastante questionáveis.

QUADRO 1. Síndromes e sintomas funcionais da atualidade e a correspondente especialidade

\begin{tabular}{|ll|}
\hline Gastroenterologia & Síndrome do intestino irritável, dispepsia funcional não ulcerosa \\
\hline Ginecologia & Síndrome pré-menstrual, dor pélvica crônica \\
\hline Reumatologia & Fibromialgia \\
\hline Cardiologia & Dor torácica não cardíaca \\
\hline Pneumologia & Síndrome da hiperventilação \\
\hline Doenças infecciosas & Síndrome da fadiga crônica (pós-viral) \\
\hline Neurologia & Cefaléia do tipo tensional \\
\hline Imunologia, alergia & Dor facial atípica \\
\hline
\end{tabular}

Adaptado de Wolfe et al. ${ }^{20}$.

Há limitações importantes na avaliação e tratamento das síndromes funcionais somáticas. A principal é que grande parte dos pacientes não aceita o diagnóstico, dificultando o tratamento; as manifestações clínicas são muito variáveis, sendo muitas vezes diagnosticadas como ansiedade ou depressão; e muitas vezes, critérios diagnósticos complexos dificultam a sua aplicabilidade pelos médicos. Por outro lado, a tendência do médico é, com freqüência, subestimar queixas de caráter funcional e a importância dos eventos psicossociais associados. As síndromes funcionais também são um problema importante dentro da prática clínica em função da prevalência elevada e do excesso de demanda aos serviços de saúde desencadeada por esses pacientes. Elas representam um grande desafio à medicina pela frequência elevada associada à evolução crônica dos sintomas com pouca melhora ao longo dos anos.

\section{Dados de prevalência}

Os dados de prevalência variam de local para local, mas sempre são números elevados. $\mathrm{Na}$ Inglaterra, as síndromes funcionais somáticas correspondem a $20 \%$ dos atendimentos na atenção primária $^{18}$. Entre pacientes ambulatoriais ingleses ${ }^{7} \mathrm{e}$ holandeses ${ }^{12}$, as síndromes funcionais somáticas são responsáveis por respectivamente $25 \%$ e $35 \%$ dos atendimentos. Entre pacientes com, no mínimo, dez internações sucessivas na Dinamarca no espaço de oito anos, $19 \%$ eram somatizadores. Aproximadamente $75 \%$ deles começaram com o quadro antes dos 25 anos, e em torno de 1/3 tinham mais de 20 anos de doença. A mediana do número de internações foi de
22 e essas internações foram responsáveis por 3\% das internações não psiquiátricas nesse período na Dinamarca ${ }^{5}$.

Dados brasileiros mostram que a prevalência ao longo da vida, no último ano, e no último mês de transtornos somatoformes em estudo de prevalência (CID-10) em área de abrangência do Instituto de Psiquiatria (HCFMUSP) em São Paulo foi respectivamente de $6 \%, 4,2 \%$ e 3,2\% ${ }^{2}$. Estudo de prevalência de síndrome da fadiga crônica no Brasil (São Paulo) e na Inglaterra mostrou prevalências bastante similares de, respectivamente, $1,6 \%$ e $2,1 \%$, usando os critérios diagnósticos do Center for Disease Control $(C D C)^{4}$. Estudo realizado no Ambulatório Geral e Didático do Hospital das Clínicas mostrou uma frequência de síndrome funcional somática de $21 \%$ (20/95) dos pacientes ${ }^{3}$.

Os 20 pacientes apresentavam 28 diagnósticos de síndrome funcional. Do total de pacientes, 16 (80\%) eram do gênero feminino. Os diagnósticos mais freqüentes foram: cefaléias crônicas $(46,4 \%)$, dispepsia funcional não ulcerosa $(32,1 \%)$, fibromialgia $(17,9 \%)$ e síndrome do intestino irritável $(3,6 \%)$, e vários pacientes apresentavam mais de um diagnóstico de síndrome funcional somática ${ }^{3}$.

A Organização Mundial da Saúde classifica as síndromes funcionais como uma comorbidade comum e debilitante na atenção primária. Além do impacto na qualidade de vida dos pacientes, possui um impacto econômico muito grande no sistema de saúde mundial, pelas passagens freqüentes dos pacientes nos serviços de saúde e pelo grande número de exames e procedimentos realizados na tentativa de se chegar a um diagnóstico. Tem seu início no 
começo da idade adulta, sendo muito mais comum em mulheres do que em homens. Mais da metade dos pacientes com transtornos ansiosos e depressivos preenchem critérios para síndromes funcionais. Outro ponto importante é o grande comprometimento da qualidade de vida, desproporcional à gravidade dos sintomas ${ }^{20}$. Por exemplo, alguns estudos mostram que o estresse emocional associado à síndrome do intestino irritável é tão grande quanto o associado às doenças inflamatórias intestinais (retocolite ulcerativa e doença de Crohn) embora as últimas sejam muito mais graves do ponto de vista clínico ${ }^{19}$. O mesmo acontece com a síndrome da fadiga crônica em relação à insuficiência cardíaca ${ }^{10}$.

Por fim, há os problemas relacionados ao tratamento. A terapêutica convencional é ineficaz na grande maioria das vezes. E como mostrado no estudo dinamarquês ${ }^{5}$ e relatado por outros autores a duração da doença é longa sendo contada muitas vezes em décadas ${ }^{13}$. Isso gera gastos elevados sem que haja resolução do problema. No Ambulatório Geral e Didático (HCFMUSP) é comum atender uma paciente com diagnóstico de cefaléia que, após o tratamento, volta com dispepsia passando por vários outros diagnósticos ao longo dos meses ${ }^{3}$. É necessário entender melhor o que se passa com esses pacientes, muitas vezes tratando o transtorno psiquiátrico associado, sob pena de nunca se resolver o problema.

\section{Síndromes funcionais somáticas: uma síndrome ou muitas síndromes}

Há uma grande discussão em relação às síndromes funcionais somáticas. Wessely et al. ${ }^{3}$, em um artigo de revisão, discute que a existência de várias síndromes é um artefato em conseqüência do alto grau de especialização da medicina ${ }^{20}$. Cada especialista focaria sempre nos sintomas da sua especialidade, sem que houvesse uma diferença importante entre os pacientes ou seus sintomas ${ }^{21}$. Ou seja, existiria somente uma síndrome funcional somática e dependendo dos sintomas que predominarem, ela receberá o rótulo de uma especialidade.

Ele propõe três perguntas para iniciar a discussão:

1. Há sobreposição dos critérios diagnósticos das várias síndromes funcionais somáticas?

2. O paciente identificado como portador de uma síndrome, pode preencher critérios para outra síndrome?

3. As síndromes compartilham algumas características em relação ao sexo dos pacientes, a coexistência de transtornos psiquiátricos, o prognóstico, ou a resposta a um mesmo tratamento? ${ }^{20}$

Há síndromes funcionais somáticas em várias especialidades médicas. Embora haja síndromes reconhecidas pelos médicos e bastante freqüentes na prática clínica, algumas outras são bem mais raras, sendo ainda motivos de polêmicas e discussões. A aceitação de grande parte dessas síndromes também varia entre os países. Por exemplo, a fibromialgia é um diagnóstico bastante freqüente no Brasil diferente da síndrome da fadiga crônica, que por outro lado, é bastante aceita na Europa e Estados Unidos. De uma maneira geral, os dados parecem apontar para uma baixa especificidade dos sintomas e grande sobreposição dos quadros funcionais entre si com um mesmo sintoma fazendo parte do critério diagnóstico de várias síndromes.

Os critérios diagnósticos de várias síndromes incluem a presença de vários sintomas em comum. $\mathrm{O}$ Quadro 2 mostra os critérios diagnósticos da síndrome da fadiga crônica, o Quadro 3 os critérios de exclusão; - Quadro 4 os critérios para diagnóstico da cefaléia do tipo tensional, o Quadro 5 o da síndrome do intestino irritável, e o Quadro 6 o da fibromialgia. Vários sintomas repetidos estão presentes nos critérios diagnósticos das várias síndromes.

QUADRO 2. Critérios diagnósticos para síndrome da fadiga crônica ${ }^{6}$

Fadiga avaliada clinicamente, mas medicamente inexplicada, com duração mínima de seis meses, que seja:

- De início recente

- Não resultante de esforço físico

- Não substancialmente aliviada pelo repouso

- Associa-se com substancial redução nos níveis prévios de atividades

Ocorrência de quatro ou mais dos seguintes sintomas:

- $\quad$ Prejuízo subjetivo da memória

- Odinofagia

- Linfonodos dolorosos

- Mialgia

- Artralgia

- Cefaléia

- Sono não reparador

- Mal-estar pós exercício físico com duração superior a 24 horas 
QUADRO 3. Critérios de exclusão para síndrome da fadiga crônica

- Doença médica ativa, não resolvida ou suspeita ou

- Depressão psicótica, melancólica ou bipolar (mas não depressão não complicada), transtornos psicóticos, demência, anorexia ou bulimia nervosa ou

- Abuso de álcool ou outras substâncias ilícitas ou

- Obesidade importante

QUADRO 4. Critérios diagnósticos para a cefaléia do tipo tensional episódica infrequente ${ }^{17}$

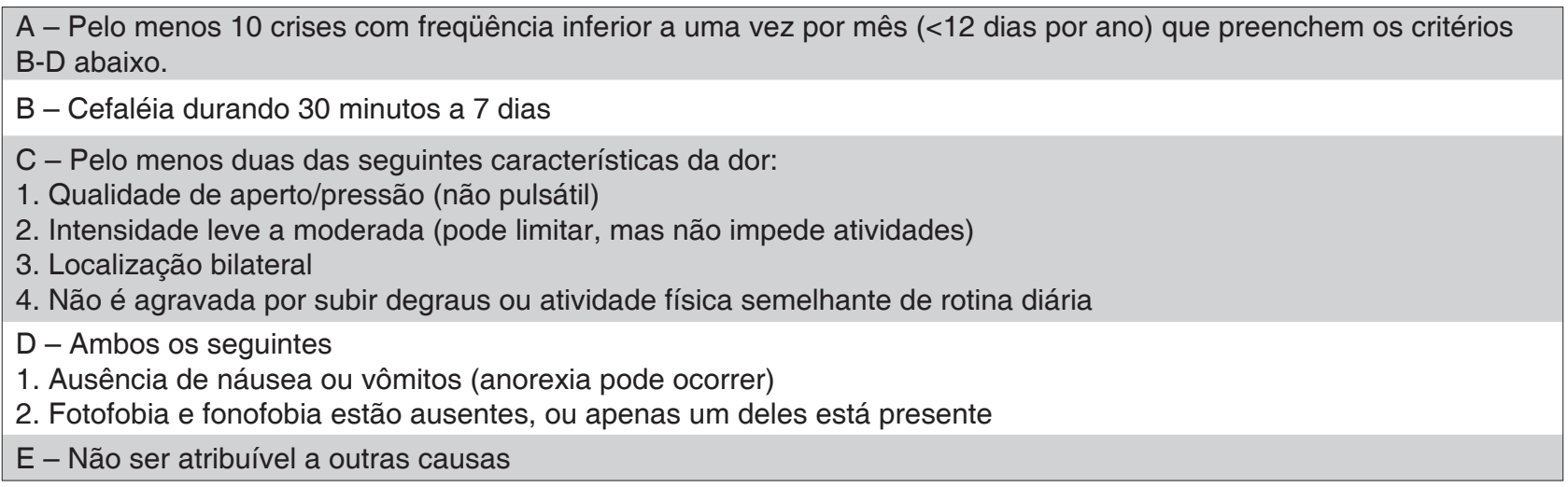

QUADRO 5. Critérios Diagnósticos para a fibromialgia²2

A. Dor generalizada nos quatro quadrantes do corpo por um mínimo de 3 meses.

B. Sensação dolorosa ao exame físico de pelo menos 11 de 18 pontos dolorosos (tender points) à digitopressão em músculo, junção tendão-músculo, coxim gorduroso ou bursa, distribuídos em todo o corpo:

- 2 em cada braço (1. tendão supraespinado; 2. ponto $2 \mathrm{~cm}$ distal do epicôndilo lateral do cotovelo);

- 1 em cada lado do tórax (junção costocondral da 2.a costela);

- 2 em cada perna (1. posterior à bursa trocantérica do fêmur; 2. coxim gorduroso medial do joelho);

- 2 em cada lado da cabeça e do pescoço (1. inserção do músculo suboccipital; 2. coluna cervical baixa, no espaço intertransverso anterior $\mathrm{C} 5-\mathrm{C} 7$ );

- 1 em cada ombro (ponto medial da borda superior do músculo trapézio);

- 1 em cada nádega (região lateral superior, no músculo).

*Pontos Controles: 1- meio da testa; 2- Face dorsal do antebraço; 3- Unha do polegar; 4-Músculos da região anterior da coxa

* Os pontos controles não podem ser dolorosos
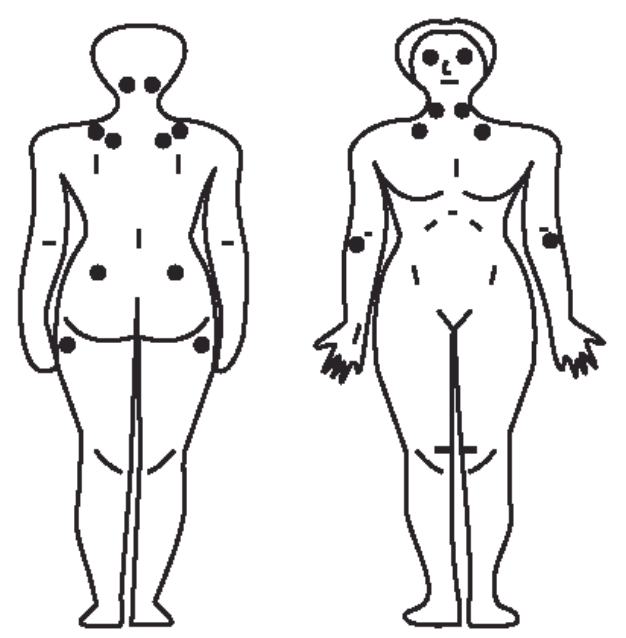
Pilan LA, Benseñor IM. Síndromes funcionais somáticas.

QUADRO 6. Critérios de ROMA III para Síndrome do Intestino Irritável'

Dor ou desconforto* abdominal durando pelo menos 3 meses (não necessariamente consecutivas) com início nos últimos 6 meses, associada com pelo menos 2 dos seguintes sintomas:

1. Alívio após evacuação, e/ou

2. Início associado com alteração na freqüência das evacuações, e/ou

3. Início associado com alteração na aparência / forma das fezes

*Desconforto significa uma sensação desconfortável, mas não descrita como dolorosa

Sintomas cumulativos que contribuem para o diagnóstico de SII:

1. Freqüência alterada das evacuações (mais de 03 evacuações por dia ou menos de 03 por semana)

2. Fezes com consistência alterada

3. Alteração do padrão de evacuação (esforço excessivo, urgência, sensação de evacuação incompleta)

4. Eliminação de muco

5. Flatulência ou sensação de distensão abdominal

O diagnóstico de um distúrbio funcional intestinal presume a ausência de uma explicação estrutural ou bioquímica para os sintomas.

Alguns sintomas aparecem como parte dos critérios diagnósticos de várias síndromes: estudos mostram que a distensão abdominal aparece como critério diagnóstico em oito síndromes, dor abdominal em seis, cefaléia em seis e fadiga em oito $^{20,21}$.

Um paciente pode preencher critérios para mais de uma síndrome. Os sintomas mais intensos podem definir uma síndrome, mas perguntando por outros sintomas, é comum observar que o mesmo paciente pode ter vários diagnósticos, embora a intensidade dos sintomas possa variar.

Todas as síndromes funcionais somáticas apresentam algumas características comuns: são mais freqüentes em mulheres do que em homens; freqüentemente há co-morbidade das várias síndromes com a presença de transtornos psiquiátricos e há evidências de uma alteração comum do funcionamento do sistema nervoso central em todas as síndromes funcionais somáticas, talvez mediadas por vias serotoninérgicas. Alguns autores discutem a presença de antecedentes de abuso sexual na infância, e já foi observada essa associação para a dor pélvica crônica, a síndrome do intestino irritável, a síndrome pré-menstrual, a fibromialgia e a síndrome da fadiga crônica ${ }^{20}$.

Outra semelhança importante é o difícil relacionamento médico-paciente muito frequente nesses casos. É comum o paciente relatar que "ninguém resolve o meu problema", o que gera estresse para o médico. Muitos pacientes exigem a realização de exames e o encaminhamento para um especialista. Entretanto, esta medida pode ser prejudicial para o tratamento do paciente, porque cada vez ele exigirá exames mais complexos criando um círculo vicioso sem fim, somado ao fato de que, em cada 20 exames pedidos, um pode ter um resultado falso-positivo que demandará mais exames para que o seu esclarecimento. Não existem exames subsidiários, tanto de laboratório como de imagem, que tenham utilidade diagnóstica para as várias síndromes, exceto quando outras enfermidades estiverem presentes concomitantemente ou somente como parte do diagnóstico diferencial. A necessidade da realização de exames complementares depende das peculiaridades de cada caso. Orientações ao paciente referentes à doença, e aos possíveis sintomas que a acompanham podem trazer uma contribuição substancial para a melhora da sua qualidade de vida.

Por último, é importante discutir as semelhanças no tratamento, que freqüentemente inclui o uso de anti-depressivos, de terapia cognitivocomportamental ou de outros tipos de terapia. Em grande parte dos casos é necessário que esse tipo de paciente seja tratado por um médico clínico com formação em psiquiatria que possa lidar com os dois aspectos das síndromes funcionais somáticas.

É importante salientar que muitos pacientes com síndromes funcionais se encontram preocupados e contrariados ao procurar auxílio médico. Esse sentimento surge pela cronicidade dos sintomas, pela não resolução ou cura com vários tratamentos já tentados anteriormente e pelo grande volume de exames que já fizeram ao longo da vida. Os pacientes podem ser enfáticos em suas queixas, e até agressivos. O médico deve estar atento na abordagem diagnóstica e no uso dos critérios diagnósticos para não ser induzido ao erro nessa delicada relação médico-paciente. Consultas breves e freqüentes têm demonstrado melhor benefício, do que consultas espaçadas e longas. É fundamental que o médico, na consulta, explique para o paciente que os sintomas são reais e 
comuns na prática clínica, identificando os fatores desencadeantes, predisponentes e perpetuadores. Deve-se dar oportunidade para o paciente discutir seus problemas pessoais e familiares e estimulálo a retornar às atividades habituais. Portanto, não se trata de passar o paciente com síndrome funcional somática do clínico para o psiquiatra, e sim de orientar esse paciente a procurar um médico generalista que "seja capaz de uma abordagem mais ampla com suporte da psiquiatria em vez de encaminhar o paciente dependendo dos sintomas que predominam a um diferente especialista".

Pilan LA, Benseñor IM. Functional somatic syndromes. Rev Med (São Paulo). 2008 out.dez.;87(4):238-44.

\begin{abstract}
Functional somatic syndromes are very frequent disorders in the frontier between the Internal Medicine and Psychiatry. Although, they are not severe disorders, they are associated with significant distress and excessive expenditure in the medical health system. Prevalence of these conditions is high, but they are still a cause of controversy and debate in relation to diagnosis and treatment.
\end{abstract}

KEY WORDS: Quality of life. Psychophysiologic disorders.

\title{
REFERÊNCIAS
}

1. Kirmayer LJ, Robbins JM. Functional somatic syndromes. In: Kirmayer LJ, Robbins JM, editors. Current concepts of somatization: research and clinical perspectives. Washington, DC: American Psychiatric Press; 1991. p.79-106.

2. Mayou R, Farmer A. ABC of psychological medicine: functional somatic symptoms and syndromes. BMJ. 2002;325(7358):265-8.

3. Wessely S, Nimnuan C, Sharpe M. Functional somatic syndromes: one or many? Lancet. 1999;354:936-9.

4. Sharpe M, Mayou R, Bass C. Concepts, theories and terminology. In: Mayou R, Bass C, Sharpe M, editors. Treatment of functional somatic symptoms. Oxford: Oxford University Press; 1995. p.3-16.

5. Kirmayer LJ, Robbins JM. Conclusion: prospects for research and clinical practice. In: Kirmayer LJ, Robbins JM, editors. Current concepts of somatization: research and clinical perspectives. Washington, Washington, DC: American Psychiatric Press; 1991. p.201-26.

6. Rief W, Hiller W. Somatization - future perspectives on a commom phenomenon. J Psychosom Res. 1998;44:529-36.

7. Rief W, Hiller W. Toward empirically based criteria for the classification of somatoform disorders. J Psychosom Res. 1999; 46:507-18.

8. Peveler R, Kilkenny L, Kinmonth A. Medically unexplained physical symptoms in primary care: a comparison of self-report screening questionnaires and clinical opinion. J Psychosom Res. 1997;42:245-53.

9. Van Henert A, Hengeveld M, Bolk J, Rooijmans H,
Vanderbroucke J. Psychological dioserders in relation to medical illness among patients of a general medical outpatient clinic. Psychol Med. 1993;23:167-73.

10. Hamilton J, Campos R, Creed F. Anxiety, depression and management of medically unexplained symptoms in medical clinics. J R Coll Physicians Lond. 1996;30(1):18-21.

11. Fink $P$. The use of hospitalizations by persistent somatizing patients. Psychol Med. 1992;22:173-80.

12. Andrade L, Walter EE, Gentil V, Laurenti R. Prevalence of CID-10 mental disorders in a catchment area in the city of São Paulo, Brazil. Soc Pschychiatry Psychiatr Epidemiol. 2002;37(7):316-25.

13. Cho HJ, Menezes PR, Hotopf M, Bhugra D, Wessely S. Comparative epidemiology of chronic fatigue syndrome in Brazilian and British primary care: prevalence and recognition. Br J Psychiatry. 2009;194(2):117-22.

14. Benseñor IJM. Avaliação do papel da anamnese, exame clínico e exames complementares no diagnóstico clínico de pacientes ambulatoriais [Tese livredocência]. São Paulo: Universidade de São Paulo, Faculdade de Medicina, Departamento de Clínica Médica; 2003.

15. Walker EA, Roy Byrne PP, Katon WJ, Li L, Amos $D$, Jiranek G. Psychiatric illness and irritable bowell syndrome: a comparison with inflammatory bowell disease. Am J Psychiatry. 1990;147:1656-61.

16. Komaroff A, Fagiolo L, Doolittle T, Gandek B, Gleit MA, Guerriero RT, et al. Health status in patients with chronic fatigue syndrome and in general 
Pilan LA, Benseñor IM. Síndromes funcionais somáticas.

population and disease comparison groups. Am J Med. 1996;101:281-90.

17. Potts S, Bass C. Psychological morbidity in patients with chest pain and normal or near normal coronary arteries: a long-term follow-up study. Psychol Med. 1995;24:339-47.

18. Fukuda, K, Straus, SE, Hickie, I, et al. The chronic fatigue syndrome: a comprehensive approach to its definition and study. International Chronic Fatigue Syndrome Study Group. Ann Intern Med. 1994;121:953-9.
19. The International Classification of Headache Disorders; 2nd edition. Cephalalgia. 2004;24(Suppl 1):9-160.

20. Wolfe F, Smythe HA, Yunus MB, Bennett RM, Bombardier C, Goldenberg DL, et al. The American College of Rheumatology 1990 Criteria for the Classification of Fibromyalgia. Report of Multicenter Criteria Committee. Arthritis Rheum. 1990;33(2):160-72.

21. American Gastroenterological Association. American Gastroenterological Association medical position statement: irritable bowel syndrome. Gastroenterology. 2002;123(6):2105-7. 